\title{
A Study of Inverse Problems Based on Two Kinds of Special Matrix Equations in Euclidean Space
}

\author{
Rui Huang, ${ }^{1}$ Xiaodong $\mathrm{Wu},{ }^{1}$ Ruihe Wang, ${ }^{2}$ and ${\mathrm{Hui} \mathrm{Li}^{3}}^{3}$ \\ ${ }^{1}$ College of Petroleum Engineering, China University of Petroleum, Beijing 102249, China \\ ${ }^{2}$ China National Oil and Gas Exploration and Development Corporation, Beijing 100034, China \\ ${ }^{3}$ CNPC Beijing Richfit Information Technology Co., Ltd., Beijing 100013, China
}

Correspondence should be addressed to Rui Huang; huangrui25@126.com

Received 11 January 2014; Accepted 13 April 2014; Published 26 May 2014

Academic Editor: Ricardo L. Soto Montero

Copyright (C) 2014 Rui Huang et al. This is an open access article distributed under the Creative Commons Attribution License, which permits unrestricted use, distribution, and reproduction in any medium, provided the original work is properly cited.

Two special classes of symmetric coefficient matrices were defined based on characteristics matrix; meanwhile, the expressions of the solution to inverse problems are given and the conditions for the solvability of these problems are studied relying on researching. Finally, the optimal approximation solution of these problems is provided.

\section{Introduction}

In recent years, a lot of matrix problems have been used widely in the fields of structural design, automatic control, physical, electrical, nonlinear programming and numerical calculation, for example, a matrix Eigen value problem was applied for mixed convection stability analysis in the Darcy media by Serebriiskii et al. [1] and some of the problems based on the nonskew symmetric orthogonal matrices were studied by Hamed and Bennacer in 2008 [2], but some of the matrix inverse problems still need further research in order to make it easier to discuss relevant issues. Therefore, in this paper, we studied the inverse problems of two kinds of special matrix equations based on the existing research achievements, moreover, the expressions and conditions of the matrix solutions are given by related matrix-calculation methods. Some definitions and assumptions of the inverse problem for two forms of special matrices are given in Section 2. In Sections 3 and 5 we discuss the existence and expressions of general solution based on the two classes of matrices, and in Sections 4 and 6 we prove the uniqueness of matrices for researching related inverse problems.

\section{Definitions and Assumptions of Inverse Problems for Two Forms of Special Matrices}

In order to research some inverse problems of related matrices, we give the following definitions and assumptions.
Definition 1. When $P \in R^{n \times n}, P=P^{T}=P^{-1}, A=\left(a_{i j}\right) \epsilon$ $R^{n \times n}, i, j=1,2,3, \ldots, n,(P A)^{T}=-P A$, and $a_{i j}=-a_{j i}, A$ will be called the first-class special symmetric matrix and the set of these special symmetric matrices is denoted by $A 1 R^{n \times n}$. The corresponding problems are as follows.

Problem 1. When $X, B \in R^{n \times m}, A \in A 1 R^{n \times n}$ can be obtained, so that $A X=B$.

Problem 2. When $\dot{A} \in R^{n \times n}, \widehat{A} \in S_{E}$ can be obtained, so that $\|\dot{A}-\widehat{A}\|=\min _{A \in S_{E}}\|\dot{A}-A\|$, where $S_{E}$ is the solution set of the first problem.

Definition 2. When $A=\left(a_{i j}\right) \in R^{n \times n}, i, j=1,2,3, \ldots, n$ and $a_{i j}=-a_{n+1-i, n+1-j}, A$ will be called the second-class special symmetric matrix and the set of these special symmetric matrices is denoted by $A 2 R^{n \times n}$. The corresponding problems are as follows.

Problem 1. When $X, B \in R^{n \times m}, A \in A 2 R^{n \times n}$ can be found, so that $A X=B$.

Problem 2. When $\dot{A} \in R^{n \times n}, \widehat{A} \in \widetilde{S}_{E}$ can be found, so that $\|\dot{A}-\widehat{A}\|=\min _{A \in \widetilde{S}_{E}}\|\dot{A}-A\|$, where $\widetilde{S}_{E}$ is the solution set of the first problem. 


\section{Existence and Expression of General Solutions Based on the First-Class Special Symmetric Matrix for Problem 1}

To research the structure and properties of the special symmetric matrix $A \in A 1 R^{n \times n}$, first of all, we have the following conclusion from Definition 1 .

Conclusion 1. Consider $A=\left(a_{i j}\right) \in R^{n \times n}$, and the sufficient and necessary conditions for $A \in A 1 R^{n \times n}$ are

$$
A^{T}=-A, \quad A^{T} P+P A=0 .
$$

Theorem 3. Consider $A=\left(a_{i j}\right) \in R^{n \times n}$, and the sufficient and necessary conditions for $A \in A 1 R^{n \times n}$ are

$$
A=U\left[\begin{array}{cc}
A_{11} & 0 \\
0 & A_{22}
\end{array}\right] U^{T}
$$

where $A_{11} \in A 1 R^{k \times k}, A_{22} \in A 1 R^{(n-k) \times(n-k)}$, and $U$ is an orthogonal matrix. Consider $U \in R^{n \times n}$ and $U U^{T}=I_{n}$.

Proof. Rely on the decomposition theorem of symmetric orthogonal matrix $[3,4]$. When $P$ is a symmetric orthogonal matrix, $P \in R^{n \times n}$, the $P$ can be represented as the following equation by an orthogonal matrix $U, U \in R^{n \times n}$ :

$$
P=U\left[\begin{array}{cc}
I_{k} & 0 \\
0 & -I_{n-k}
\end{array}\right] U^{T}
$$

where $I$ is identity matrix [5].

When $A \in A 1 R^{n \times n}$, we can derive from (1) and (3) the following:

$$
U^{T} A^{T} U\left[\begin{array}{cc}
I_{k} & 0 \\
0 & -I_{n-k}
\end{array}\right]+\left[\begin{array}{cc}
I_{k} & 0 \\
0 & -I_{n-k}
\end{array}\right] U^{T} A U=0 .
$$

Based on $A^{T}=-A$ and $U^{T} A U \in A 1 R^{n \times n}$, there will be

$$
U^{T} A U=\left[\begin{array}{cc}
A_{11} & A_{12} \\
-A_{12}^{T} & A_{22}
\end{array}\right]
$$

and $A=U\left[\begin{array}{cc}A_{11} & A_{12} \\ -A_{12}^{T} & A_{22}\end{array}\right] U^{T}$ can be derived from (4) and (5).

Conversely, when

$$
A=U\left[\begin{array}{cc}
A_{11} & A_{12} \\
-A_{12}^{T} & A_{22}
\end{array}\right] U^{T}
$$

$A^{T}=-A, A^{T} P+P A=0$ can be obtained, and, relying on Conclusion 1, $A \in A 1 R^{n \times n}$ can be obtained.

Theorem 4. When $X, B \in R^{n \times m}$ and the $X$ singular value decomposition is

$$
X=U_{1}\left[\begin{array}{cc}
\Sigma_{1} & 0 \\
0 & 0
\end{array}\right] V_{1}^{T}
$$

where $U_{1}=\left[\begin{array}{ll}U^{(1)} & U^{(2)}\end{array}\right] \in O R^{n \times n}, V_{1}=\left[\begin{array}{ll}V^{(1)} & V^{(2)}\end{array}\right] \epsilon$ $O R^{m \times m}$, and $\Sigma_{1}=\operatorname{diag}\left(\delta_{1}, \ldots, \delta_{r}\right)>0, r=\operatorname{rank}(X), U^{(1)} \epsilon$ $R^{n \times r}, U^{(2)} \in R^{n \times(n-r)}, V^{(1)} \in R^{m \times r}, V^{(2)} \in R^{m \times(m-r)}, O R^{n \times n}$ is the set of orthogonal matrix, and $X^{+}$is Moore-Penrose generalized inverse matrix [6-8].

The sufficient and necessary conditions for the existence of solution $A X=B$ are

$$
B^{T} X=-X^{T} B, \quad B X^{+} X=B .
$$

And the general solution is as follows:

$$
A=B X^{+}-\left(B X^{+}\right)^{T}\left(I_{n}-X X^{+}\right)+U^{(2)} G\left(U^{(2)}\right)^{T},
$$

where $G \in A 1 R^{(n-r) \times(n-r)}$.

Proof. Define

$$
\begin{aligned}
U_{1}^{T} A U_{1} & =\left[\begin{array}{ll}
A_{11} & A_{12} \\
A_{21} & A_{22}
\end{array}\right], \\
U_{1}^{T} B V_{1} & =\left[\begin{array}{ll}
B_{11} & B_{12} \\
B_{21} & B_{22}
\end{array}\right],
\end{aligned}
$$

where $A_{11} \in R^{r \times r}$ and $B_{i j}=\left(U^{(1)}\right)^{T} B V^{(1)}, i, j=1,2$.

The equation $A X=B$ can be represented as the following equation by (7) and (10):

$$
\left[\begin{array}{ll}
A_{11} & A_{12} \\
A_{21} & A_{22}
\end{array}\right]\left[\begin{array}{cc}
\Sigma_{1} & 0 \\
0 & 0
\end{array}\right]=\left[\begin{array}{ll}
B_{11} & B_{12} \\
B_{21} & B_{22}
\end{array}\right]
$$

According to (7) and (8), there will be

$$
B_{11}^{T} \Sigma_{1}=-\Sigma_{1} B_{11}, \quad B_{12}=0, \quad B_{22}=0 .
$$

Then (11) is equivalent to the following equation:

$$
\begin{aligned}
& A_{11} \Sigma_{1}=B_{11}, \\
& A_{21} \Sigma_{1}=B_{21},
\end{aligned}
$$

and $A_{11}=B_{11} \Sigma_{1}^{-1} \in A 1 R^{r \times r}$ and $A_{21}=B_{21} \Sigma_{1}^{-1}$ can be obtained from (12) and (13), so the equation $A X=B$ has solutions, $A \in A 1 R^{n \times n}$. And the general solution can be represented as the following equation:

$$
\begin{aligned}
A & =U_{1}\left[\begin{array}{cc}
B_{11} \Sigma_{1}^{-1} & -\Sigma_{1}^{-1} B_{21}^{T} \\
B_{21} \Sigma_{1}^{-1} & A_{22}
\end{array}\right] U_{1}^{T} \\
& =B X^{+}-\left(B X^{+}\right)^{T}\left(I_{n}-X X^{+}\right)+U^{(2)} G\left(U^{(2)}\right)^{T},
\end{aligned}
$$

where $G \in A 1 R^{(n-r) \times(n-r)}$

Conversely, when the equation $A X=B$ has solutions and $A \in A 1 R^{n \times n}, B X^{+} X=B$ and $X^{T} B=X^{T} A X$ can be obtained relying on the Penrose theorem $[9,10]$.

There will be

$$
B^{T} X=\left(X^{T} B\right)^{T}=\left(X^{T} A X\right)^{T}=-X^{T} A X=-X^{T} B .
$$

The equations $B^{T} X=-X^{T} B$ and $B X^{+} X=B$ are provided.

Finally the proof of Theorems 3 and 4 is completed. 
From Theorems 3 and 4 we have a corollary as follows.

Corollary 5. Consider that $X, B \in R^{n \times m}, X_{i}$ and $B_{i}(i=1,2)$, $X_{2}, B_{2} \in R^{(n-k) \times m}$, and $X_{1}, B_{1} \in R^{k \times m}$ are given by these equations:

$$
U^{T} X=\left[\begin{array}{l}
X_{1} \\
X_{2}
\end{array}\right], \quad U^{T} B=\left[\begin{array}{l}
B_{1} \\
B_{2}
\end{array}\right] .
$$

The $X_{i}$ singular value decompositions are

$$
X_{1}=U_{1}\left[\begin{array}{cc}
\Sigma_{1} & 0 \\
0 & 0
\end{array}\right] V_{1}^{T}, \quad X_{2}=U_{2}\left[\begin{array}{cc}
\Sigma_{2} & 0 \\
0 & 0
\end{array}\right] V_{2}^{T},
$$

where $U_{1}=\left[\begin{array}{ll}U_{1}^{(1)} & U_{1}^{(2)}\end{array}\right] \in O R^{k \times k}, V_{1}=\left[\begin{array}{ll}V_{1}^{(1)} & V_{1}^{(2)}\end{array}\right] \in O R^{m \times m}$, $U_{2}=\left[\begin{array}{ll}U_{2}^{(1)} & U_{2}^{(2)}\end{array}\right] \in O R^{(n-k) \times(n-k)}, V_{2}=\left[\begin{array}{ll}V_{2}^{(1)} & V_{2}^{(2)}\end{array}\right] \epsilon$ $O R^{m \times m}, \Sigma_{1}=\operatorname{diag}\left(\delta_{1}^{1}, \ldots, \delta_{r_{1}}^{1}\right)>0, \Sigma_{2}=\operatorname{diag}\left(\delta_{1}^{2}, \ldots, \delta_{r_{2}}^{2}\right)>$ $0, r_{1}=\operatorname{rank}\left(X_{1}\right), r_{2}=\operatorname{rank}\left(X_{2}\right), V_{1}^{(1)} \in R^{m \times r_{1}}, U_{2}^{(1)} \in$ $R^{(n-k) \times r_{2}}, U_{1}^{(1)} \in R^{k \times r_{1}}, V_{2}^{(1)} \in R^{m \times r_{2}}$.

The sufficient and necessary conditions for the existence of solution $A X=B$ are

$$
B_{i}^{T} X_{i}=-X_{i}^{T} B_{i}, \quad B_{i} X_{i}^{+} X_{i}=B_{i} .
$$

And the general solution is as follows:

$$
\begin{aligned}
A= & U\left[\begin{array}{cc}
A_{11} & 0 \\
0 & A_{22}
\end{array}\right] U^{T} \\
& +U\left[\begin{array}{cc}
U_{1}^{(2)} G_{1}\left(U_{1}^{(2)}\right)^{T} & 0 \\
0 & U_{2}^{(2)} G_{2}\left(U_{2}^{(2)}\right)^{T}
\end{array}\right] U^{T}, \\
A_{11}= & B_{1} X_{1}^{+}-\left(B_{1} X_{1}^{+}\right)^{T}\left(I_{k}-X_{1} X_{1}^{+}\right) \\
A_{22}= & B_{2} X_{2}^{+}-\left(B_{2} X_{2}^{+}\right)^{T}\left(I_{n-k}-X_{2} X_{2}^{+}\right),
\end{aligned}
$$

where $G_{1} \in A 1 R^{\left(k-r_{1}\right) \times\left(k-r_{1}\right)}$ and $G_{2} \in A 1 R^{\left(n-k-r_{2}\right) \times\left(n-k-r_{2}\right)}$.

The proof is completed.

\section{The Unique Solutions on the First-Class Special Symmetric Matrix for Problem 2}

Consider the following theorem.

Theorem 6. When $X, B \in R^{n \times m}, A \in R^{n \times n}$, and $X_{i}$ and $B_{i}(i=$ 1,2) are given by (16) and obey (18), Problem 2 has the unique solution $\widehat{A} \in S_{E}$.

Define

$$
U^{T} \dot{A} U=\left[\begin{array}{cc}
\dot{A}_{11} & \dot{A}_{12} \\
\dot{A}_{21} & \dot{A}_{22}
\end{array}\right], \quad \dot{A}_{11} \in R^{k \times k} .
$$

Then the unique solution $\widehat{A}$ can be represented as follows:

$$
\widehat{A}=U\left[\begin{array}{cc}
\dot{A}_{11} & 0 \\
0 & \dot{A}_{22}
\end{array}\right] U^{T},
$$

where

$$
\begin{aligned}
\widehat{A}_{11}= & A_{11}^{0}+0.5 \\
& \cdot\left(I_{k}-X_{1} X_{1}^{+}\right)\left(\dot{A}_{11}-\dot{A}_{11}^{T}\right)\left(I_{k}-X_{1} X_{1}^{+}\right), \\
\widehat{A}_{22}= & A_{22}^{0}+0.5 \\
& \cdot\left(I_{n-k}-X_{2} X_{2}^{+}\right)\left(\dot{A}_{22}-\dot{A}_{22}^{T}\right)\left(I_{n-k}-X_{2} X_{2}^{+}\right) .
\end{aligned}
$$

$A_{0}$ is defined by the following equation:

$$
\begin{aligned}
A_{0} & =U\left[\begin{array}{cc}
A_{11}^{0} & 0 \\
0 & A_{22}^{0}
\end{array}\right] U^{T}, \\
A_{11}^{0} & =B_{1} X_{1}^{+}-\left(B_{1} X_{1}^{+}\right)^{T}\left(I_{k}-X_{1} X_{1}^{+}\right), \\
A_{22}^{0} & =B_{2} X_{2}^{+}-\left(B_{2} X_{2}^{+}\right)^{T}\left(I_{n-k}-X_{2} X_{2}^{+}\right) .
\end{aligned}
$$

Finally $S_{E}$ can represent the following set by Theorem 4:

$$
\begin{aligned}
S_{E}= & A_{0} \\
& +U\left[\begin{array}{cc}
U_{1}^{(2)} G_{2}\left(U_{1}^{(2)}\right)^{T} & 0 \\
0 & U_{2}^{(2)} G_{2}\left(U_{2}^{(2)}\right)^{T}
\end{array}\right] U^{T} .
\end{aligned}
$$

Consider $G_{1} \in A 1 R^{\left(k-r_{1}\right) \times\left(k-r_{1}\right)}$ and $G_{2} \in A 1 R^{\left(n-k-r_{2}\right) \times\left(n-k-r_{2}\right)}$.

Proof. Define these following equations:

$$
\begin{aligned}
& U_{1}^{T}\left(\dot{A}_{11}-A_{11}^{0}\right) U_{1}=\left[\begin{array}{ll}
\dot{A}_{11}^{1} & \dot{A}_{12}^{1} \\
\dot{A}_{21}^{1} & \dot{A}_{22}^{1}
\end{array}\right], \\
& U_{2}^{T}\left(\dot{A}_{22}-A_{22}^{0}\right) U_{2}=\left[\begin{array}{ll}
\dot{A}_{11}^{2} & \dot{A}_{12}^{2} \\
\dot{A}_{21}^{2} & \dot{A}_{22}^{2}
\end{array}\right],
\end{aligned}
$$

where $\dot{A}_{11}^{1} \in R^{r_{1} \times r_{1}}$ and $\dot{A}_{22}^{1} \in R^{r_{2} \times r_{2}}$.

Because of $A \in S_{E}$ we can obtain

$$
\begin{aligned}
A & =A_{0}+U\left[\begin{array}{cc}
U_{1}^{(2)} G_{1}\left(U_{1}^{(2)}\right)^{T} & 0 \\
0 & U_{2}^{(2)} G_{2}\left(U_{2}^{(2)}\right)^{T}
\end{array}\right] U^{T} \\
& =A_{0}+U\left[\begin{array}{cc}
U_{1}\left[\begin{array}{cc}
0 & 0 \\
0 & G_{1}
\end{array}\right] U_{1}^{T} & 0 \\
0 & U_{2}\left[\begin{array}{cc}
0 & 0 \\
0 & G_{2}
\end{array}\right] U_{2}^{T}
\end{array}\right] U^{T},
\end{aligned}
$$

$$
\begin{aligned}
& \|\dot{A}-A\|^{2}=\left\|\left[\begin{array}{cc}
U_{1}^{(2)} G_{2}\left(U_{1}^{(2)}\right)^{T} & 0 \\
0 & U_{2}^{(2)} G_{2}\left(U_{2}^{(2)}\right)^{T}
\end{array}\right]\right\|^{2} \\
& =\left\|\left[\begin{array}{cc}
U_{1}^{(2)} G_{2}\left(U_{1}^{(2)}\right)^{T} & 0 \\
0 & U_{2}^{(2)} G_{2}\left(U_{2}^{(2)}\right)^{T}
\end{array}\right]\right\|^{2}
\end{aligned}
$$




$$
\begin{aligned}
= & \left\|\dot{A}_{12}\right\|^{2}+\left\|\dot{A}_{21}\right\|^{2} \\
& +\left\|\left[\begin{array}{cc}
0 & 0 \\
0 & G_{1}
\end{array}\right]-U_{1}^{T}\left(\dot{A}_{11}-A_{11}^{0}\right) U_{1}\right\|^{2} \\
& +\left\|\left[\begin{array}{cc}
0 & 0 \\
0 & G_{2}
\end{array}\right]-U_{2}^{T}\left(\dot{A}_{22}-A_{22}^{0}\right) U_{2}\right\|^{2} .
\end{aligned}
$$

Finally,

$$
\begin{aligned}
\|\dot{A}-A\|^{2}= & \left\|\dot{A}_{12}\right\|^{2}+\left\|\dot{A}_{21}\right\|^{2}+\left\|\dot{A}_{11}^{1}\right\|^{2} \\
& +\left\|\dot{A}_{12}^{1}\right\|^{2}+\left\|\dot{A}_{21}^{1}\right\|^{2}+\left\|\dot{A}_{11}^{2}\right\|^{2}+\left\|\dot{A}_{12}^{2}\right\|^{2} \\
& +\left\|\dot{A}_{21}^{2}\right\|^{2}+\left\|G_{1}-\dot{A}_{22}^{1}\right\|^{2}+\left\|G_{2}-\dot{A}_{22}^{2}\right\|^{2} .
\end{aligned}
$$

When $H=-H^{T}, H \in R^{n \times m}$ and $E \in R^{n \times m}$, we can obtain:

$$
\left\|E-\frac{E-E^{T}}{2}\right\| \leq\|E-H\|
$$

$$
C=\left[\begin{array}{c}
U_{1}^{(2)}\left(U_{1}^{(2)}\right)^{T}\left(\dot{A}_{11}-\dot{A}_{11}^{T}\right) U_{1}^{(2)}\left(U_{1}^{(2)}\right)^{T} \\
0
\end{array}\right.
$$

and $\widehat{A}=U\left[\begin{array}{cc}\dot{A}_{11} & 0 \\ 0 & \dot{A}_{22}\end{array}\right] U^{T}$ can be obtained because of $U_{1}^{(2)}\left(U_{1}^{(2)}\right)^{T}=I_{n-k}-X_{1} X_{1}^{+}$and $U_{2}^{(2)}\left(U_{2}^{(2)}\right)^{T}=I_{k}-X_{2} X_{2}^{+}$.

Finally, rely on the optimal approximation [11,12], and $S_{E}$ is a closed convex set; Problem 2 has the unique solution $\widehat{A} \epsilon$ $S_{E}, S_{E} \in R^{n \times n}$, so that $\|\dot{A}-\widehat{A}\|=\min _{A \in \widetilde{S}_{E}}\|\dot{A}-A\|$.

The proof is completed.

\section{Conditions for the Existence and}

\section{Expression of General Solutions}

Based on the Second-Class Special

\section{Symmetric Matrix for Problem 1}

Consider the following theorem.

Theorem 7. Consider that $A=\left(a_{i j}\right) \in R^{n \times n}$, and the sufficient and necessary conditions for $A \in A 2 R^{n \times n}$ are

$$
A=U\left[\begin{array}{cc}
0 & F_{1} \\
F_{2} & 0
\end{array}\right] U^{T},
$$

where $U$ is an orthogonal matrix. $U \in R^{n \times n}$ and $U U^{T}=I_{n}$.

When $n=2 k+1$, define

$$
U=\frac{1}{\sqrt{2}}\left[\begin{array}{ccc}
I_{k} & 0 & I_{k} \\
0 & \sqrt{2} & 0 \\
H_{k} & 0 & -H_{k}
\end{array}\right] .
$$

When $G_{1}=0.5 \cdot\left(\dot{A}_{22}^{1}-\left(\dot{A}_{22}^{1}\right)^{T}\right)$ and $G_{2}=0.5 \cdot\left(\dot{A}_{22}^{2}-\left(\dot{A}_{22}^{2}\right)^{T}\right)$, so that $\|\dot{A}-A\|=\min _{A \in S_{E}}$,

$$
\begin{aligned}
& G_{1}=0.5 \cdot\left(U_{1}^{(2)}\right)^{T}\left(\dot{A}_{11}-\dot{A}_{11}^{T}-2 A_{11}^{0}\right) U_{1}^{(2)}, \\
& G_{2}=0.5 \cdot\left(U_{2}^{(2)}\right)^{T}\left(\dot{A}_{22}-\dot{A}_{22}^{T}-2 A_{22}^{0}\right) U_{2}^{(2)}
\end{aligned}
$$

can be derived from (26) and

$$
\left(U_{1}^{(2)}\right)^{T} A_{11}^{0} U_{1}^{(2)}=0, \quad\left(U_{2}^{(2)}\right)^{T} A_{22}^{0} U_{2}^{(2)}=0
$$

can be obtained according to (17), (18), and (24).

Therefore,

$$
\begin{aligned}
& G_{1}=0.5 \cdot\left(U_{1}^{(2)}\right)^{T}\left(\dot{A}_{11}-\dot{A}_{11}^{T}\right) U_{1}^{(2)}, \\
& G_{2}=0.5 \cdot\left(U_{2}^{(2)}\right)^{T}\left(\dot{A}_{22}-\dot{A}_{22}^{T}\right) U_{2}^{(2)} .
\end{aligned}
$$

Relying on (27) the solution $\widehat{A}$ can be provided as follows:

$$
\widehat{A}=A_{0}+\frac{1}{2} U C U^{T},
$$

where
When $n=2 k$, define

$$
\begin{gathered}
U=\frac{1}{\sqrt{2}}\left[\begin{array}{cc}
I_{k} & I_{k} \\
H_{k} & -H_{k}
\end{array}\right], \\
H_{k}=\left[\begin{array}{cccc}
0 & \cdots & 0 & 1 \\
0 & \cdots & 1 & 0 \\
\cdots & \cdots & \cdots & \cdots \\
1 & \cdots & 0 & 0
\end{array}\right], \quad H_{k}^{T} H_{k}=I_{k}, \quad H_{k}^{T}=H_{k},
\end{gathered}
$$

$F_{1} \in A 2 R^{(n-k) \times k}, F_{2} \in A 2 R^{k \times(n-k)}$.

Proof. First relying on the definition and property of the matrix $A \in A 2 R^{n \times n}, A 2 R^{n \times n}$ can represent the following set. When $n=2 k+1$,

$$
A=\left[\begin{array}{ccc}
C_{1} & u & C_{2} H_{k} \\
-v^{T} & 0 & v^{T} H_{k} \\
-H_{k} C_{2} & -H_{k} u & -H_{k} C_{1} H_{k}
\end{array}\right],
$$

$C_{1}, C_{2} \in R^{k \times k}$ and $u, v \in R^{k}$.

When $n=2 k$,

$$
A=\left[\begin{array}{cc}
C_{1} & C_{2} H_{k} \\
-H_{k} C_{2} & -H_{k} C_{1} H_{k}
\end{array}\right],
$$

$C_{1}, C_{2} \in R^{k \times k}$. 
We first discuss the topic $n=2 k+1$.

From (37) and (39),

$$
\begin{gathered}
U^{T} A U=\frac{1}{2}\left[\begin{array}{ccc}
I_{k} & 0 & H_{k} \\
0 & \sqrt{2} & 0 \\
I_{k} & 0 & -H_{k}
\end{array}\right] C\left[\begin{array}{ccc}
I_{k} & 0 & I_{k} \\
0 & \sqrt{2} & 0 \\
H_{k} & 0 & -H_{k}
\end{array}\right], \\
C=\left[\begin{array}{ccc}
C_{1} & u & C_{2} H_{k} \\
-v^{T} & 0 & v^{T} H_{k} \\
-H_{k} C_{2} & -H_{k} u & -H_{k} C_{1} H_{k}
\end{array}\right], \\
U^{T} A U=\left[\begin{array}{ccc}
0 & 0 & C_{1}-C_{2} \\
0 & 0 & -\sqrt{2} v^{T} \\
C_{1}+C_{2} & \sqrt{2} u & 0
\end{array}\right] .
\end{gathered}
$$

Define $F_{1}=\left[\begin{array}{c}C_{1}-C_{2} \\ -\sqrt{2} v^{T}\end{array}\right], F_{2}=\left[\begin{array}{ll}C_{1}+C_{2} & \sqrt{2} u\end{array}\right]$.

According to $C_{1}, C_{2} \in R^{k \times k}$ and $u, v \in R^{k}$, we have $F_{1} \in$ $R^{(n-k) \times k}$ and $F_{2} \in R^{k \times(n-k)}$, and

$$
A=U U^{T} A U U^{T}=U\left[\begin{array}{cc}
0 & F_{1} \\
F_{2} & 0
\end{array}\right] U^{T}
$$

can be obtained from (42).

Conversely, when $A=U\left[\begin{array}{cc}0 & F_{1} \\ F_{2} & 0\end{array}\right] U^{T}, F_{1} \in R^{(n-k) \times k}, F_{2} \in$ $R^{k \times(n-k)}, A=-H_{n} A H_{n}$ can be obtained and $A \in A 2 R^{n \times n}$ will be provided.

When $n=2 k$, in the same way, we can prove the above theorem.

The proof is completed.

Theorem 8. When $X, B \in R^{n \times m}, A \in A 2 R^{n \times n}$, and $X_{i}$ and $B_{i}(i=1,2)$ are given by $(16)$, the sufficient and necessary conditions for the existence of solution $A X=B$ are

$$
B_{i} X_{i}^{+} X_{i}=B_{i} .
$$

And the general solution is as follows:

$$
\begin{gathered}
A=U\left[\begin{array}{cc}
0 & A_{12} \\
A_{21} & 0
\end{array}\right] U^{T}, \\
A_{12}=B_{1} X_{2}^{+}+M_{1}\left(I_{k}-X_{2} X_{2}^{+}\right), \\
A_{21}=B_{2} X_{1}^{+}+M_{2}\left(I_{n-k}-X_{1} X_{1}^{+}\right),
\end{gathered}
$$

where

$$
\begin{aligned}
& U=\frac{1}{\sqrt{2}}\left[\begin{array}{ccc}
I_{k} & 0 & I_{k} \\
0 & \sqrt{2} & 0 \\
H_{k} & 0 & -H_{k}
\end{array}\right], \quad n=2 k+1, \\
& U=\frac{1}{\sqrt{2}}\left[\begin{array}{cc}
I_{k} & I_{k} \\
H_{k} & -H_{k}
\end{array}\right], \quad n=2 k .
\end{aligned}
$$

Meanwhile $M_{1} \in R^{(n-k) \times k}, M_{2} \in R^{k \times(n-k)}$, and according to (17) we can obtain $X_{i}$ singular value decompositions:

$$
X_{1}=U_{1}\left[\begin{array}{cc}
\Sigma_{1} & 0 \\
0 & 0
\end{array}\right] V_{1}^{T}, \quad X_{2}=U_{2}\left[\begin{array}{cc}
\Sigma_{2} & 0 \\
0 & 0
\end{array}\right] V_{2}^{T} .
$$

Proof. Relying on Theorem 7, if there is $A \in A 2 R^{n \times n}$, it will have

$$
A=U\left[\begin{array}{cc}
0 & F_{1} \\
F_{2} & 0
\end{array}\right] U^{T}, \quad F_{1} \in A 2 R^{(n-k) \times k}, \quad F_{2} \in A 2 R^{k \times(n-k)} .
$$

Because $U$ is an orthogonal matrix and $U U^{T}=I_{n}$,

$$
\|A X-B\|^{2}=\left\|U^{T} A U U^{T} X-U^{T} B\right\|^{2} .
$$

We can derive from (16), (48), and (49)

$$
\begin{aligned}
\|A X-B\|^{2} & =\left\|\left[\begin{array}{cc}
0 & F_{1} \\
F_{2} & 0
\end{array}\right]\left[\begin{array}{l}
X_{1} \\
X_{2}
\end{array}\right]-\left[\begin{array}{l}
B_{1} \\
B_{2}
\end{array}\right]\right\|^{2} \\
& =\left\|F_{1} X_{2}-B_{1}\right\|^{2}+\left\|F_{2} X_{1}-B_{2}\right\|^{2} .
\end{aligned}
$$

It will be known that, when $X \in R^{n \times k}, B \in R^{n \times k}$, and $\operatorname{rank}(X)=r$ [13], the sufficient and necessary conditions for the existence of solution $A X=B$ are $B X^{+} X=B$ and the general solution is

$$
A=B X^{+}+M\left(I_{m}-X X^{+}\right), \quad M \in R^{n \times m} .
$$

There will be

$$
\begin{array}{ll}
F_{1}=B_{1} X_{2}^{+}+M_{1}\left(I_{k}-X_{2} X_{2}^{+}\right), & M_{1} \in R^{(n-k) \times k} ; \\
F_{2}=B_{2} X_{1}^{+}+M_{2}\left(I_{n-k}-X_{1} X_{1}^{+}\right), & M_{2} \in R^{k \times(n-k)} .
\end{array}
$$

The general solution can obtain

$$
\begin{gathered}
A=U\left[\begin{array}{cc}
0 & A_{12} \\
A_{21} & 0
\end{array}\right] U^{T}, \\
A_{12}=B_{1} X_{2}^{+}+M_{1}\left(I_{k}-X_{2} X_{2}^{+}\right), \\
A_{21}=B_{2} X_{1}^{+}+M_{2}\left(I_{n-k}-X_{1} X_{1}^{+}\right) .
\end{gathered}
$$

The sufficient and necessary conditions for the existence of solution $A X=B$ are $B_{i} X_{i}^{+} X_{i}=B_{i}$.

The proof is completed.

\section{The Unique Solutions on the Second-Class Special Symmetric Matrix for Problem 2}

Consider the following theorem.

Theorem 9. When, $B \in R^{n \times m}, A \in R^{n \times n}$, and $X_{i}$ and $B_{i}(i=$ $1,2)$ are given by (16) and (17) and obey (18), Problem 2 has the unique solution $\widehat{A} \in \widetilde{S}_{E}$.

The unique solution $\widehat{A}$ can be represented as follows:

$$
\widehat{A}=U C U^{T},
$$

C

$$
=\left[\begin{array}{cc}
0 & B_{1} X_{2}^{+}+A_{12}\left(I_{k}-X_{2} X_{2}^{+}\right) \\
B_{2} X_{1}^{+}+A_{21}\left(I_{n-k}-X_{1} X_{1}^{+}\right) & 0
\end{array}\right],
$$


where

$$
A_{0}=U\left[\begin{array}{cc}
0 & B_{1} X_{2}^{+} \\
B_{2} X_{1}^{+} & 0
\end{array}\right] U^{T}
$$

$n=2 k+1, \dot{A}_{2} \in A 2 R^{n \times n}:$

$$
\begin{aligned}
& A_{12}=0.5 \cdot\left[\begin{array}{ccc}
I_{k} & 0 & H_{k} \\
0 & \sqrt{2} & 0
\end{array}\right]\left(\dot{A}_{2}-A_{0}\right)\left[\begin{array}{c}
I_{k} \\
0 \\
-H_{k}
\end{array}\right], \\
& A_{21}=0.5 \cdot\left[\begin{array}{lll}
I_{k} & 0 & -H_{k}
\end{array}\right]\left(\dot{A}_{2}-A_{0}\right)\left[\begin{array}{cc}
I_{k} & 0 \\
0 & \sqrt{2} \\
H_{k} & 0
\end{array}\right] ;
\end{aligned}
$$

$n=2 k, \dot{A}_{2} \in A 2 R^{n \times n}$ :

$$
\begin{aligned}
& A_{12}=0.5 \cdot\left[\begin{array}{ll}
I_{k} & H_{k}
\end{array}\right]\left(\dot{A}_{2}-A_{0}\right)\left[\begin{array}{c}
I_{k} \\
-H_{k}
\end{array}\right], \\
& A_{21}=0.5 \cdot\left[\begin{array}{ll}
I_{k} & -H_{k}
\end{array}\right]\left(\dot{A}_{2}-A_{0}\right)\left[\begin{array}{c}
I_{k} \\
H_{k}
\end{array}\right] .
\end{aligned}
$$

From Theorem $9 \widetilde{S}_{E}$ can be represented as follows:

$$
\begin{gathered}
\widetilde{S}_{E}=A_{0}+U\left[\begin{array}{cc}
0 & M_{1}\left(I_{k}-X_{2} X_{2}^{+}\right) \\
M_{2}\left(I_{n-k}-X_{1} X_{1}^{+}\right) & 0
\end{array}\right] U^{T} \\
M_{1} \in R^{(n-k) \times k}, \quad M_{2} \in R^{k \times(n-k)} .
\end{gathered}
$$

Proof. Defining this following equation:

$$
U^{T}\left(\dot{A}_{2}-A_{0}\right) U=\left[\begin{array}{ll}
A_{11} & A_{12} \\
A_{21} & A_{22}
\end{array}\right],
$$

where $n=2 k+1, \dot{A}_{2} \in A 2 R^{n \times n}$,

$$
\begin{aligned}
& A_{11}=0.5 \cdot\left[\begin{array}{ccc}
I_{k} & 0 & H_{k} \\
0 & \sqrt{2} & 0
\end{array}\right]\left(\dot{A}_{2}-A_{0}\right), \\
& A_{12}=0.5 \cdot\left[\begin{array}{ccc}
I_{k} & 0 & H_{k} \\
0 & \sqrt{2} & 0
\end{array}\right]\left(\dot{A}_{2}-A_{0}\right)\left[\begin{array}{c}
I_{k} \\
0 \\
-H_{k}
\end{array}\right], \\
& A_{21}=0.5 \cdot\left[\begin{array}{lll}
I_{k} & 0 & -H_{k}
\end{array}\right]\left(\dot{A}_{2}-A_{0}\right)\left[\begin{array}{cc}
I_{k} & 0 \\
0 & \sqrt{2} \\
H_{k} & 0
\end{array}\right], \\
& A_{22}=0.5 \cdot\left[\begin{array}{lll}
I_{k} & 0 & -H_{k}
\end{array}\right]\left(\dot{A}_{2}-A_{0}\right)\left[\begin{array}{cc}
I_{k} \\
0 \\
-H_{k}
\end{array}\right] ;
\end{aligned}
$$

$n=2 k, \dot{A}_{2} \in A 2 R^{n \times n}:$

$$
\begin{aligned}
& A_{11}=0.5 \cdot\left[\begin{array}{ll}
I_{k} & H_{k}
\end{array}\right]\left(\dot{A}_{2}-A_{0}\right)\left[\begin{array}{c}
I_{k} \\
H_{k}
\end{array}\right], \\
& A_{12}=0.5 \cdot\left[\begin{array}{ll}
I_{k} & H_{k}
\end{array}\right]\left(\dot{A}_{2}-A_{0}\right)\left[\begin{array}{c}
I_{k} \\
-H_{k}
\end{array}\right], \\
& A_{21}=0.5 \cdot\left[\begin{array}{ll}
I_{k} & -H_{k}
\end{array}\right]\left(\dot{A}_{2}-A_{0}\right)\left[\begin{array}{c}
I_{k} \\
H_{k}
\end{array}\right], \\
& A_{22}=0.5 \cdot\left[\begin{array}{ll}
I_{k} & -H_{k}
\end{array}\right]\left(\dot{A}_{2}-A_{0}\right)\left[\begin{array}{c}
I_{k} \\
-H_{k}
\end{array}\right] .
\end{aligned}
$$

For $A \in \widetilde{S}_{E}$ the following equation can be obtained:

$$
\begin{gathered}
A=A_{0}+U C U^{T}, \\
C=\left[\begin{array}{cc}
0 & M_{1}\left(I_{k}-X_{2} X_{2}^{+}\right) \\
M_{2}\left(I_{n-k}-X_{1} X_{1}^{+}\right) & 0
\end{array}\right] .
\end{gathered}
$$

When $\dot{A} \in R^{n \times n}$ is known and relies on the definition of $A \in A 2 R^{n \times n}$, there will be the unique set $\dot{A}_{1}=\left(\bar{a}_{i j}\right) \epsilon$ $R^{n \times n}\left(\bar{a}_{i j}=\bar{a}_{(n+1-i),(n+1-j)}\right)$ and $\dot{A}_{2} \in A 2 R^{n \times n}$ to make the following equations true:

$$
\begin{array}{cl}
\dot{A}=\dot{A}_{1}+\dot{A}_{2}, & \left(\dot{A}_{1}, \dot{A}_{2}\right)=0, \\
\dot{A}_{2}=0.5 \cdot\left(\dot{A}+H_{n} \dot{A} H_{n}\right), & \dot{A}_{1}=0.5 \cdot\left(\dot{A}-H_{n} \dot{A} H_{n}\right),
\end{array}
$$$$
\|\dot{A}-A\|^{2}=\left\|\dot{A}_{1}+\dot{A}_{2}-A\right\|^{2}=\left\|\dot{A}_{1}\right\|^{2}+\left\|\dot{A}_{2}-A\right\|^{2} .
$$

From (60), (63), and (65) we obtain

$$
\begin{aligned}
& \|\dot{A}-A\|^{2}=\left\|\dot{A}_{1}\right\|^{2}+\left\|\begin{array}{c}
U\left[\begin{array}{cc}
A_{11} & A_{12} \\
A_{21} & A_{22}
\end{array}\right] U^{T} \\
-U C U^{T}
\end{array}\right\|^{2} \\
& =\left\|\dot{A}_{1}\right\|^{2}+\left\|\left[\begin{array}{cc}
A_{11} & A_{12} \\
A_{21} & A_{22} \\
-C
\end{array}\right]\right\|^{2}, \\
& C=\left[\begin{array}{cc}
0 & M_{1}\left(I_{k}-X_{2} X_{2}^{+}\right) \\
M_{2}\left(I_{n-k}-X_{1} X_{1}^{+}\right) & 0
\end{array}\right] \text {. }
\end{aligned}
$$

Equation (67) can be known from (17) and (64) as follows:

$$
\begin{gathered}
U_{2}^{(2)}\left(U_{2}^{(2)}\right)^{T}=I_{k}-X_{2} X_{2}^{+}, \\
U_{1}^{(2)}\left(U_{1}^{(2)}\right)^{T}=I_{n-k}-X_{1} X_{1}^{+}, \\
\left(U_{2}^{(2)}\right)^{T} U_{2}^{(2)}=I_{k-r_{2}}
\end{gathered}
$$




$$
\begin{gathered}
\left(U_{1}^{(2)}\right)^{T} U_{1}^{(2)}=I_{n-k-r_{1}}, \\
\left(U_{2}^{(2)}\right)^{T} U_{2}^{(1)}=0, \\
\left(U_{1}^{(2)}\right)^{T} U_{1}^{(1)}=0, \\
\|\dot{A}-A\|^{2} \\
=\left\|\dot{A}_{1}\right\|^{2}+\left\|A_{11}\right\|^{2}+\left\|A_{22}\right\|^{2} \\
+\left\|A_{12}-M_{1} U_{2}^{(2)}\left(U_{2}^{(2)}\right)^{T}\right\|^{2}+\left\|A_{12}-M_{2} U_{1}^{(2)}\left(U_{1}^{(2)}\right)^{T}\right\|^{2} \\
=\left\|\dot{A}_{1}\right\|^{2}+\left\|A_{11}\right\|^{2}+\left\|A_{22}\right\|^{2}+\left\|A_{12} U_{2}^{(1)}\right\|^{2}+\left\|A_{21} U_{1}^{(1)}\right\|^{2} \\
+\left\|A_{12} U_{2}^{(2)}-M_{1} U_{2}^{(2)}\right\|^{2}+\left\|A_{21} U_{1}^{(2)}-M_{2} U_{1}^{(2)}\right\|^{2} .
\end{gathered}
$$

Therefore, $\|\dot{A}-A\|=\min _{A \in \widetilde{S}_{E}}$ is equivalent to

$$
\begin{gathered}
\left\{\begin{array}{l}
\left\|A_{12} U_{2}^{(2)}-M_{1} U_{2}^{(2)}\right\|=\min _{M_{1} \in R^{(n-k) \times k}} \\
\left\|A_{12} U_{1}^{(2)}-M_{2} U_{1}^{(2)}\right\|=\min _{M_{2} \in R^{k \times(n-k)}}
\end{array}\right. \\
\Longrightarrow\left\{\begin{array}{l}
A_{12} U_{2}^{(2)}-M_{1} U_{2}^{(2)}=0 \\
A_{12} U_{1}^{(2)}-M_{2} U_{1}^{(2)}=0
\end{array}\right. \\
\Longrightarrow\left\{\begin{array}{l}
A_{12} U_{2}^{(2)}=M_{1} U_{2}^{(2)} \\
A_{12} U_{1}^{(2)}=M_{2} U_{1}^{(2)},
\end{array}\right.
\end{gathered}
$$

and $A_{12}\left(I_{k}-X_{2} X_{2}^{+}\right)=M_{1}\left(I_{k}-X_{2} X_{2}^{+}\right), A_{21}\left(I_{n-k}-X_{1} X_{1}^{+}\right)=$ $M_{2}\left(I_{n-k}-X_{1} X_{1}^{+}\right)$can be obtained.

From the above results and (63), the solution $\widehat{A}\left(\widehat{A} \in \widetilde{S}_{E}\right)$ can be represented as follows:

$$
\widehat{A}=U C U^{T},
$$

C

$$
=\left[\begin{array}{cc}
0 & B_{1} X_{2}^{+}+A_{12}\left(I_{k}-X_{2} X_{2}^{+}\right) \\
B_{2} X_{1}^{+}+A_{21}\left(I_{n-k}-X_{1} X_{1}^{+}\right) & 0
\end{array}\right] .
$$

Finally, because $\widetilde{S}_{E}$ is a closed convex set, Problem 2 has the unique solution $\widehat{A} \in \widetilde{S}_{E}, \widetilde{S}_{E} \in R^{n \times n}$, so that $\|\dot{A}-\widehat{A}\|=$ $\min _{A \in \tilde{S}_{E}}\|\dot{A}-A\|$.

The proof is completed.

\section{Conflict of Interests}

The authors declare that there is no conflict of interests regarding the publication of this paper.

\section{Acknowledgment}

The authors are grateful to the referee for the useful comments and valuable suggestions.

\section{References}

[1] I. Serebriiskii, R. Castelló-Cros, A. Lamb, E. A. Golemis, and E. Cukierman, "Fibroblast-derived 3D matrix differentially regulates the growth and drug-responsiveness of human cancer cells," Matrix Biology, vol. 27, no. 6, pp. 573-585, 2008.

[2] H. B. Hamed and R. Bennacer, "Analytical development of disturbed matrix eigenvalue problem applied to mixed convection stability analysis in Darcy media," Comptes Rendus Mécanique, vol. 336, no. 8, pp. 656-663, 2008.

[3] C. W. H. Lam, "Non-skew symmetric orthogonal matrices with constant diagonals," Discrete Mathematics, vol. 43, no. 1, pp. 6578, 1983.

[4] X. Liu, B. M. Nguelifack, and T.-Y. Tam, "Unitary similarity to a complex symmetric matrix and its extension to orthogonal symmetric Lie algebras," Linear Algebra and Its Applications, vol. 438, no. 10, pp. 3789-3796, 2013.

[5] J. D. Hill, "Polynomial identities for matrices symmetric with respect to the symplectic involution," Journal of Algebra, vol. 349 , no. 1, pp. 8-21, 2012.

[6] M. A. Rakha, "On the Moore-Penrose generalized inverse matrix," Applied Mathematics and Computation, vol. 158, no. 1, pp. 185-200, 2004.

[7] H. Yanai, "Some generalized forms a least squares $g$-inverse, minimum norm $g$-inverse, and Moore-Penrose inverse matrices," Computational Statistics \& Data Analysis, vol. 10, no. 3, pp. 251-260, 1990.

[8] J. K. Baksalary and O. M. Baksalary, "Particular formulae for the Moore-Penrose inverse of a columnwise partitioned matrix," Linear Algebra and Its Applications, vol. 421, no. 1, pp. 16-23, 2007.

[9] I. Lindner and G. Owen, "Cases where the Penrose limit theorem does not hold," Mathematical Social Sciences, vol. 53, no. 3, pp. 232-238, 2007.

[10] I. Lindner and M. Machover, "L.S. Penrose's limit theorem: proof of some special cases," Mathematical Social Sciences, vol. 47, no. 1, pp. 37-49, 2004.

[11] G. Papakonstantinou, "Optimal polygonal approximation of digital curves," Signal Processing, vol. 8, no. 1, pp. 131-135, 1985.

[12] R. Ferretti, "Convergence of semidiscrete approximations to optimal control problems in hilbert spaces: a counterexample," Systems \& Control Letters, vol. 27, no. 2, pp. 125-128, 1996.

[13] Y. Li, F. Zhang, W. Guo, and J. Zhao, "Solutions with special structure to the linear matrix equation $\mathrm{AX}=\mathrm{B}$," Computers \& Mathematics with Applications, vol. 61, no. 2, pp. 374-383, 2011. 


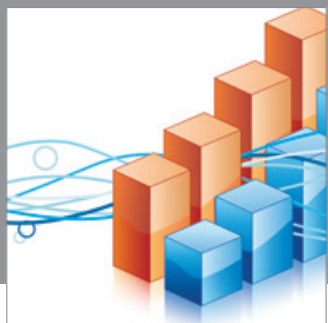

Advances in

Operations Research

mansans

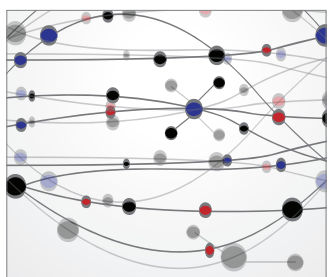

The Scientific World Journal
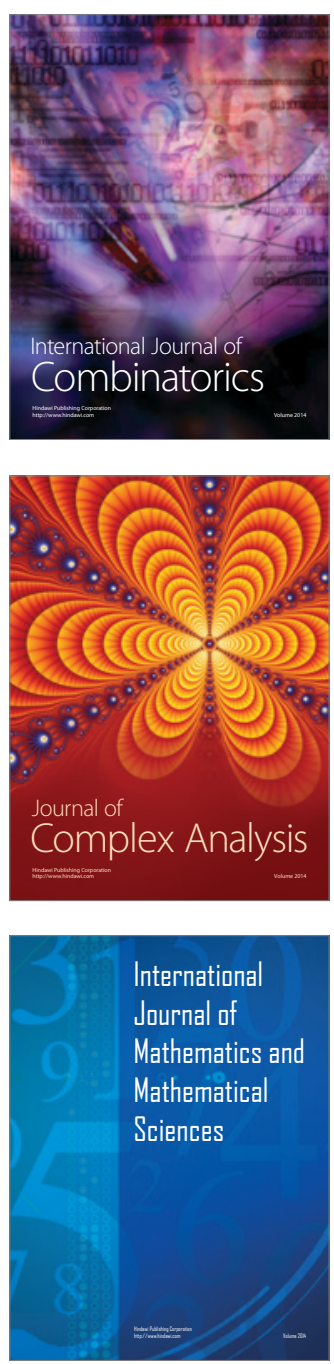
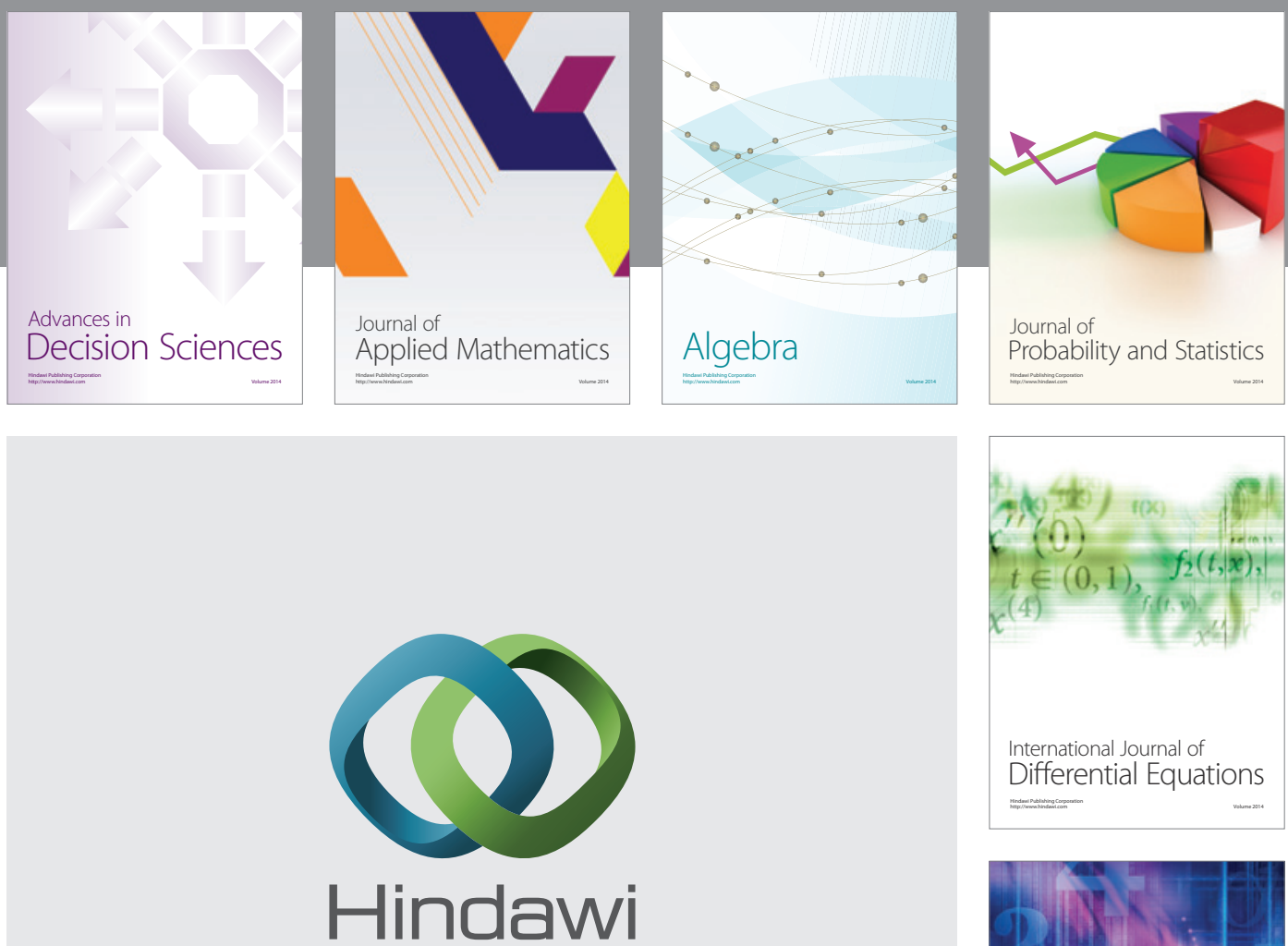

Submit your manuscripts at http://www.hindawi.com
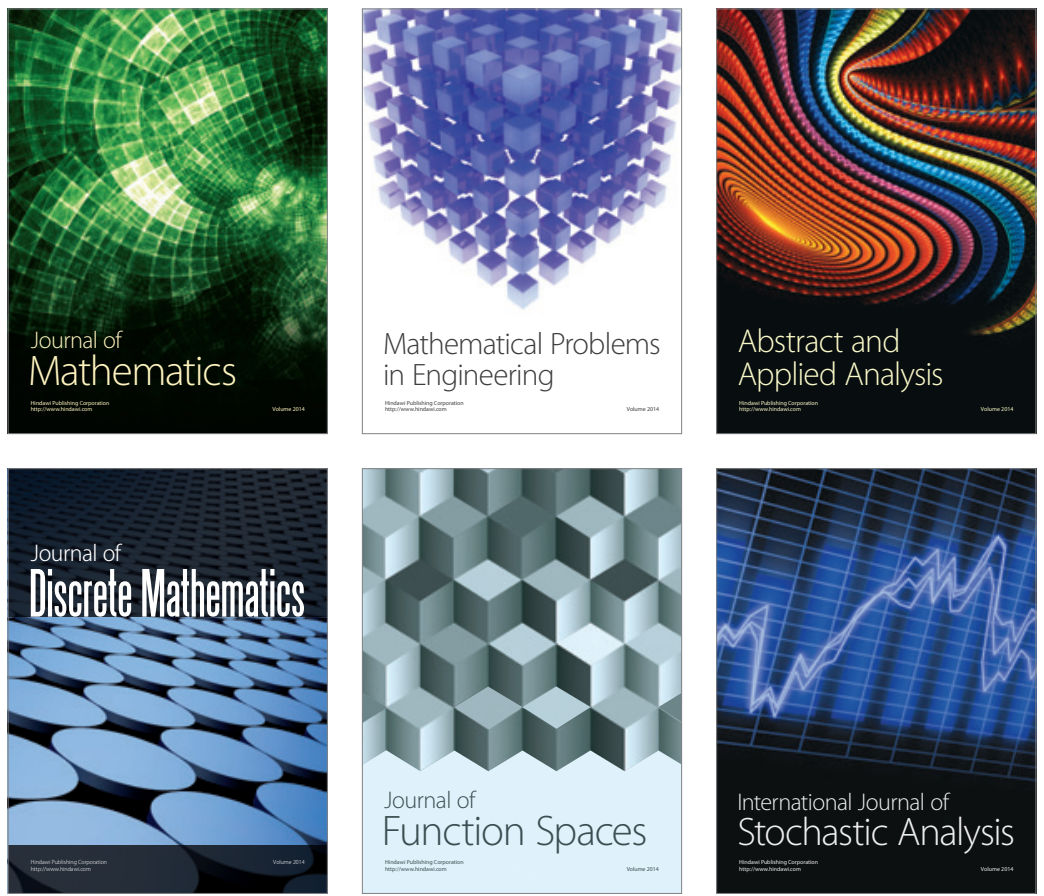

Journal of

Function Spaces

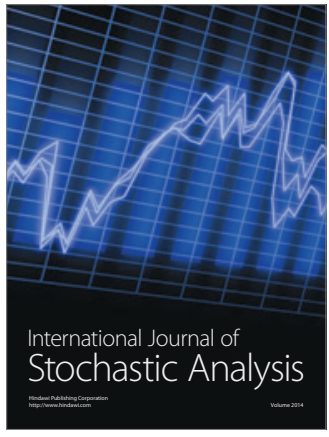

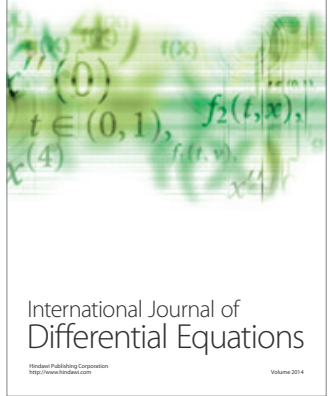
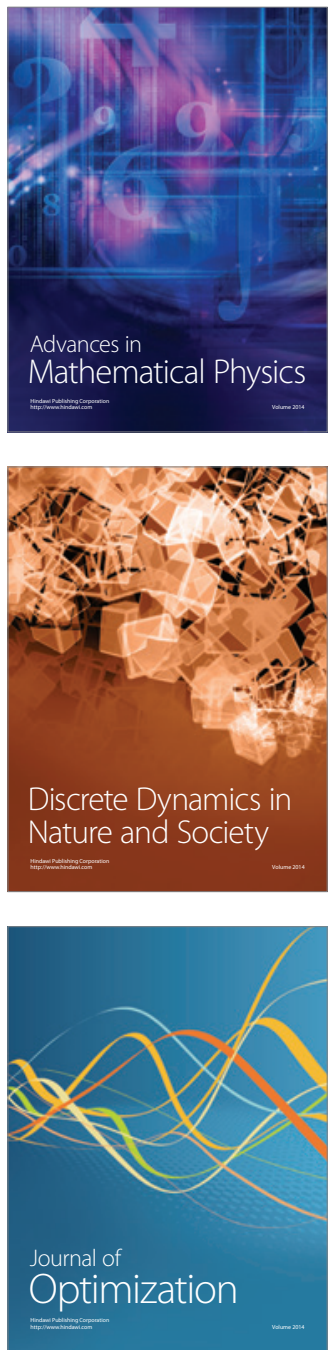